\title{
Dilemas éticos ante la posibilidad de padecer la enfermedad de Alzheimer u otras demencias. Estudio exploratorio
}

\author{
Asunción Álvarez-del Río ${ }^{*}$ y Ma. Luisa Marván² \\ ${ }^{1}$ Universidad Nacional Autónoma de México, Facultad de Medicina, Departamento de Psiquiatría y Salud Mental, Ciudad de México; ${ }^{2}$ Universidad \\ Veracruzana, Instituto de Investigaciones Psicológicas, Veracruz. México
}

\section{Resumen}

Introducción: En México se han realizado esfuerzos para incrementar la comprensión de la enfermedad de Alzheimer y otras demencias, así como para mejorar la atención de los pacientes con estas enfermedades y la de sus cuidadores. Sin embargo, no se ha investigado el interés de las personas por la toma de decisiones ante la posibilidad de vivir con dichas enfermedades y enfrentar los dilemas éticos implicados. Objetivo: Conocer las opiniones de adultos maduros sobre algunos dilemas éticos ante la posibilidad de vivir con enfermedad de Alzheimer u otras demencias. Métodos: Estudio observacional, transversal y correlacional. Los participantes contestaron un cuestionario autoaplicable. Resultados: 134 adultos maduros respondieron el cuestionario; 70.9 \% había pensado en la posibilidad de padecer alguna demencia y la mayoría desearía conocer su diagnóstico, aproximadamente la mitad comunicó a sus familiares su voluntad sobre tratamientos médicos en el futuro, 39.6 \% no aprobaba alimentar artificialmente a un paciente que no puede comer ni decidir y $37.3 \%$ sí lo aprobaba. Conclusiones: Hay interés en las decisiones anticipadas ante la posibilidad de padecer demencia. Para responder a las interrogantes al respecto es importante continuar investigando sobre el tema, así como para resolver algunos dilemas éticos y promover el uso de la voluntad anticipada.

PALABRAS CLAVE: Enfermedad de Alzheimer. Demencia. Adultos maduros. Dilemas éticos.

\section{Ethical dilemmas in the face of the possibility of suffering from Alzheimer's disease or other dementias. An exploratory study}

\begin{abstract}
Introduction: In Mexico, efforts have been made to increase understanding of Alzheimer's disease and other dementias, as well as to improve the care of patients with these diseases and that of their caregivers. However, people's interest in making decisions and facing the ethical dilemmas regarding the possibility of living with mental diseases has not been investigated. Objective: To know the opinions of mature adults on some ethical dilemmas related to the possibility of living with Alzheimer's disease or other dementias. Methods: Observational, cross-sectional, correlational study. Participants answered a self-administered questionnaire. Results: 134 mature adults answered the questionnaire; $70.9 \%$ had thought about the possibility of suffering from some dementia and the vast majority would like to know their diagnosis; approximately half the participants had informed their families of their wishes about medical treatment in the future; $39.6 \%$ did not approve artificially feeding a patient who can no longer eat or decide; $37.3 \%$ did approve this. Conclusions: There is interest in advance decisions in the face of the possibility of suffering from dementia. To answer unanswered questions in this regard, it is important for research on the subject to continue, as well as to solve some ethical dilemmas and promote the use of advance directives.
\end{abstract}

KEY WORDS: Alzheimer's disease. Dementia. Mature adults. Ethical dilemmas.

Correspondencia:

*Asunción Álvarez-del Río

E-mail: asun57@gmail.com

DOI: 10.24875/GMM.20000848
Gac Med Mex. 2021;157:418-424

Disponible en PubMed

www.gacetamedicademexico.com

CC BY-NC-ND (http://creativecommons.org/licenses/by-nc-nd/4.0/). 


\section{Introducción}

El mejoramiento de los servicios de salud y los grandes avances científicos y tecnológicos han permitido incrementar considerablemente la esperanza de vida, pero numerosas personas viven en una situación de dependencia que se considera indigna, como sucede con la enfermedad de Alzheimer (EA), la forma más común de demencia. Igual que otras demencias, causa el declive progresivo de las funciones cognitivas, deterioro del control emocional y de la conducta social, con lo que destruyen la personalidad, las relaciones y la habilidad para decidir. ${ }^{1} \mathrm{La}$ $E A$, igual que otras demencias, es una condición que algunas personas temen más que el cáncer. ${ }^{2}$

La EA puede presentarse a cualquier edad alcanzada la adultez, pero más comúnmente en la edad avanzada; dado el envejecimiento de la población, cada vez más personas podrán padecerla. ${ }^{3}$ En 2019, según la Organización Mundial de la Salud, en el orbe había 50 millones de personas con demencia. ${ }^{4}$ En México se calcula que para 2050 habrá 3.5 millones de adultos mayores con alguna demencia. ${ }^{5}$ La probabilidad de padecer EA al pasar los 65 años es de uno entre cada 20 individuos; en mayores de 80 , de uno entre cada cinco. ${ }^{3}$

Se han establecido tres etapas para la EA: leve, moderada y grave. Durante la primera, el enfermo sigue realizando actividades y toma decisiones; en la moderada, es incapaz del cuidado personal, lo que aumenta la dependencia; en la grave, el deterioro y la dependencia son totales y se pierden las características psicológicas de la persona. ${ }^{6}$ La duración de la EA puede variar entre siete y 10 años, pero hay pacientes que viven más. Como sucede con otras demencias, la EA no tiene curación y solo puede retrasarse su avance.

El diagnóstico de la EA es esencialmente clínico basado en el deterioro cognitivo, una vez descartadas otras causas. Los estudios de neuroimagen y los biomarcadores apoyan el diagnóstico, el cual es concluyente con el estudio histopatológico después del fallecimiento. ${ }^{1}$ El diagnóstico temprano de una demencia es buen momento para realizar un documento de voluntad anticipada (DVA), pues la persona conserva la capacidad para decidir. ${ }^{7}$

En el más reciente informe de la Alzheimer's Disease International, se reporta una encuesta a casi 70 mil personas (público general, profesionales de la salud, enfermos y cuidadores) de 155 países. Casi
$95 \%$ pensó que podría desarrollar demencia y $78 \%$ temía dicha enfermedad; $85 \%$ de las personas con demencia dijo que su opinión no contaba en su atención. ${ }^{8}$

Los casos de EA genéticamente determinados son minoría. ${ }^{9}$ Otros factores parecen influir en la aparición del padecimiento como enfermedad vascular cerebral, hipertensión arterial, diabetes tipo 2, sobrepeso y colesterol. ${ }^{10}$ También se han identificado algunos factores protectores: dieta, actividad física y ejercicios cognitivos. ${ }^{11}$ Mientras algunas personas adquieren hábitos para prevenir la $\mathrm{EA}$, unas más confían en que se descubran tratamientos para prevenirla o curarla $^{12}$ y otras quieren tener el control si enferman.

Se ha reportado el Advance Care Planning (planificación del cuidado) y los DVA por personas que no quieren vivir con demencia avanzada. ${ }^{13,14}$ En México puede suscribirse un DVA manifestando decisiones legales como la suspensión (o no iniciación) de tratamientos que prolongan la vida y solicitar cuidados paliativos. ${ }^{15}$

En México se han realizado esfuerzos importantes de investigación sobre demencias y para la atención de pacientes y sus cuidadores, ${ }^{3,16}$ considerando los principios y dilemas éticos implicados; ${ }^{1}$ sin embargo, no se ha investigado el interés de las personas acerca de tomar decisiones ante la posibilidad de vivir con demencia. Iniciamos una línea de investigación para estudiar este aspecto, con la cual esperamos contribuir a la discusión de las decisiones sobre los cuidados y tratamientos de pacientes en fases avanzadas.

El objetivo de este primer estudio fue conocer las opiniones de adultos maduros sobre el interés de saber su diagnóstico si padecen demencia, y sobre la alimentación e hidratación artificiales para pacientes en etapa avanzada, así como saber si han comunicado por anticipado su voluntad respecto a tratamientos futuros.

\section{Métodos}

Las comisiones de ética y de investigación de la Facultad de Medicina de la UNAM aprobaron este estudio.

La muestra estuvo constituida por 134 adultos maduros de la Ciudad de México con estudios universitarios. La mayoría fueron reclutados en lugares públicos y otros en casas de día para adultos mayores. El criterio de inclusión fue tener entre 50 y 75 años, saber que la EA se caracteriza por pérdida 
paulatina de la memoria y que es una enfermedad progresiva sin cura (se preguntó qué sabían de la EA). El criterio de exclusión fue tener diagnóstico de demencia o presentar alguna condición física o mental que comprometiera su comprensión. La tasa de aceptación fue de $92 \%$.

Los participantes dieron su consentimiento informado y contestaron un cuestionario elaborado para esta investigación y previamente probado. Además de algunos datos sociodemográficos, se exploró lo siguiente:

- Si los participantes conocían a alguien cercano que padeciera EA u otras demencias y si estaban implicados directamente en su cuidado.

- Si habían pensado en la posibilidad de padecer EA u otras demencias.

- Si querían conocer el diagnóstico con las opciones de respuesta "sí", "no", "no estoy seguro", con la justificación de su respuesta.

- Si sus familiares conocían su voluntad sobre sus tratamientos si padecieran otra enfermedad y no pudieran decidir debido a la demencia.

- Si estaban o no de acuerdo con la alimentación e hidratación artificiales para pacientes con EA u otras demencias sin capacidad de decidir. Nuevamente, las opciones de respuesta fueron "sí", "no", "no estoy seguro", con la justificación de su respuesta.

Se realizaron tablas de contingencia con la prueba de chi cuadrada para conocer la asociación entre las variables (resultados significativos con una $p \leq 0.05$ ) y un análisis de contenido de las preguntas abiertas para generar categorías. La confiabilidad fue entre 0.84 y 1 utilizando el índice kappa de Cohen.

\section{Resultados}

Se encuestó a 134 personas de entre 50 y 77 años. La Tabla 1 describe sus características sociodemográficas. La mitad de los participantes $(n=68)$ conocía a alguien cercano con demencia; de estos, $37.3 \%$ $(n=25)$ estuvo implicado directamente en su cuidado. La mayoría de los participantes $(n=95,70.9 \%)$ había pensado que podría padecer EA u otras demencias; de ellos, la mayoría $(n=56,58.9 \%$ ) dijo conocer a alguien cercano que ha padecido demencia. Al contrario, la mayor parte de los que no lo habían pensado ( $n=27,69.2 \%$ ) no conocían a alguien cercano con demencia (resultados estadísticamente significativos, $\chi^{2}=7.69, p=0.006$ ).
Tabla 1. Características sociodemográficas de los participantes

\begin{tabular}{|c|c|c|}
\hline \multirow{2}{*}{ Edad en años, media $\pm \mathrm{DE}$} & \multicolumn{2}{|c|}{$59.9 \pm 7.8$} \\
\hline & $\mathrm{n}$ & $\%$ \\
\hline $\begin{array}{l}\text { Sexo } \\
\text { Mujer } \\
\text { Hombre }\end{array}$ & $\begin{array}{l}82 \\
52\end{array}$ & $\begin{array}{l}61.2 \\
38.8\end{array}$ \\
\hline $\begin{array}{l}\text { Religión } \\
\text { Católica o derivada del cristianismo } \\
\text { Otra } \\
\text { Ninguna }\end{array}$ & $\begin{array}{l}109 \\
3 \\
22\end{array}$ & $\begin{array}{c}81.3 \\
2.2 \\
16.4\end{array}$ \\
\hline $\begin{array}{l}\text { Religiosidad } \\
\text { Muy importante } \\
\text { Importante } \\
\text { Poco importante } \\
\text { Nada importante }\end{array}$ & $\begin{array}{l}30 \\
48 \\
31 \\
23\end{array}$ & $\begin{array}{l}22.7 \\
36.4 \\
23.5 \\
17.4\end{array}$ \\
\hline $\begin{array}{l}\text { Con quien vive } \\
\text { Pareja } \\
\text { Pareja e hijos } \\
\text { Hijos } \\
\text { Solo } \\
\text { Otro familiar } \\
\text { Cuidador }\end{array}$ & $\begin{array}{c}34 \\
41 \\
21 \\
22 \\
14 \\
1\end{array}$ & $\begin{array}{c}25.4 \\
30.6 \\
15.7 \\
16.4 \\
10.4 \\
0.7\end{array}$ \\
\hline
\end{tabular}

A la pregunta de si desearía conocer el diagnóstico de padecer demencia, la gran mayoría contestó afirmativamente $(n=126,94 \%)$. De los ocho restantes encuestados, cinco respondieron que no y tres no estaban seguros. En la Tabla 2 se muestran las categorías en las que fueron clasificadas las explicaciones. La respuesta más común de querer saber su diagnóstico fue "prepárarse", seguida de "toma de decisiones" y "búsqueda de opciones terapéuticas". La respuesta principal de no querer saber o no estar seguro fue "doloroso".

El $50.7 \%$ de los participantes aseguró que sus familiares saben su voluntad en caso de tener demencia en etapa avanzada y que necesitaran decidir sus tratamientos; $88.1 \%$ de ellos $(n=59)$ dijo que conocen su voluntad por pláticas; $6 \%(n=4)$ disponía de un DVA notariado; el resto con un documento no oficial.

Sobre la opinión de los participantes de alimentar e hidratar artificialmente a pacientes con demencia que ya no pueden comer y sin capacidad de decidir, $39.6 \%(n=53)$ no estuvo de acuerdo, mientras que $38.8 \%(n=52)$ sí y el resto no estaba seguro. En la Tabla 3 se muestran las categorías de las explicaciones. El porcentaje de sujetos que estuvieron de acuerdo fue semejante al de individuos que no. La mayoría de los que estuvieron de acuerdo consideran que "es un deber"; la respuesta de los que no estuvieron de acuerdo fue "futilidad terapéutica". Para la 
Tabla 2. Razones por las que los participantes quisieran o no que se les comunicara su diagnóstico de enfermedad de Alzheimer u otra demencia

\begin{tabular}{|c|c|c|c|}
\hline Categoría de respuesta & $n$ & $\%$ & Ejemplo de respuesta \\
\hline \multicolumn{4}{|c|}{ Participantes que sí quisieran saber su diagnóstico } \\
\hline $\begin{array}{l}\text { Es un derecho } \\
\text { Respuestas que denotan la exigencia de lo que se } \\
\text { considera éticamente merecido. }\end{array}$ & 9 & 7.4 & $\begin{array}{l}\text { Porque todas las personas tienen derecho a saber qué les } \\
\text { ocurre en su cuerpo. }\end{array}$ \\
\hline $\begin{array}{l}\text { Prepararse } \\
\text { Respuestas que indican que desearían saber el } \\
\text { diagnóstico para realizar acciones o buscar las } \\
\text { condiciones psicológicas óptimas para enfrentar la } \\
\text { enfermedad. }\end{array}$ & 38 & 31.1 & $\begin{array}{l}\text { Para prepararse psicológicamente y recibir apoyo de la } \\
\text { familia. }\end{array}$ \\
\hline $\begin{array}{l}\text { Búsqueda de opciones terapéuticas } \\
\text { Respuestas que expresan interés en saber el diagnóstico } \\
\text { para tener la oportunidad de recibir ayuda terapéutica } \\
\text { que mejore su calidad de vida y retarde el avance de } \\
\text { la enfermedad. Algunas respuestas se refieren a la } \\
\text { prevención del deterioro. }\end{array}$ & 28 & 23.0 & $\begin{array}{l}\text { Para cuidarme y atenderme. Porque podría luchar por } \\
\text { disminuir lo más que se pueda el daño. }\end{array}$ \\
\hline $\begin{array}{l}\text { Toma de decisiones } \\
\text { Respuestas que indican interés en saber el diagnóstico } \\
\text { para ser ellos quienes definan cómo quieren ser tratados } \\
\text { cuando ya no puedan expresar su voluntad. }\end{array}$ & 29 & 23.8 & Para poder decidir sobre mi futuro. \\
\hline $\begin{array}{l}\text { Entendimiento de la situación } \\
\text { Respuestas que expresan interés en vivir con el } \\
\text { conocimiento y la comprensión de lo que implica la } \\
\text { enfermedad. }\end{array}$ & 15 & 12.3 & Para saber qué está pasando. \\
\hline $\begin{array}{l}\text { Aprovechar el tiempo } \\
\text { Quisieran saber el diagnóstico para sacar el máximo } \\
\text { beneficio del tiempo de vida que les quedaría antes de } \\
\text { deteriorarse por la enfermedad. }\end{array}$ & 3 & 2.5 & Para hacer las cosas que quiero hacer antes de irme. \\
\hline Total & 122 & 100 & \\
\hline \multicolumn{4}{|c|}{ Participantes que no quisieran saber su diagnóstico o no están seguros de querer o no } \\
\hline $\begin{array}{l}\text { Doloroso } \\
\text { Respuestas que expresan que no quisieran saberlo } \\
\text { porque les causaría mucha aflicción. }\end{array}$ & 5 & 71.4 & Para qué, es muy triste. \\
\hline $\begin{array}{l}\text { Negación } \\
\text { Respuestas que denotan que preferirían vivir como si no } \\
\text { estuvieran enfermos. }\end{array}$ & 2 & 28.6 & Mejor no saber esas cosas y disfrutar. \\
\hline Total & 7 & 100 & \\
\hline
\end{tabular}

mayoría de los sujetos que no estaban seguros, dependería de la "voluntad del paciente".

No hubo asociaciones significativas entre estar de acuerdo o no con la alimentación e hidratación artificiales y el hecho de conocer a alguien cercano con demencia o haber participado en su cuidado.

\section{Discusión}

Este primer estudio de una línea de investigación explora las opiniones de adultos maduros sobre algunos dilemas éticos de la atención de personas con demencias y el interés de mantener el control de padecer EA u otras demencias; también constituye el primero que aborda este aspecto en México. Se enfocó en adultos maduros entre 50 y 75 años.

Como la edad aumenta la probabilidad de padecer demencia, no sorprende que aproximadamente la mitad de los participantes conociera a alguien cercano con EA u otras demencias. Esto pudo haber influido en que la mayoría haya pensado en padecerla, aunque en porcentaje menor $(70.9 \%)$ al del 
Tabla 3. Razones por las que los participantes están o no de acuerdo con la alimentación e hidratación artificiales para pacientes con demencia que ya no tengan la capacidad de decidir

\begin{tabular}{l|l|l|l|} 
Categoría de respuesta & $n$ & $\%$ & Ejemplo de respuesta
\end{tabular}

Participantes que no estuvieron de acuerdo en la hidratación y alimentación artificial

Futilidad terapéutica

Cuando consideran que la hidratación y alimentación

artificiales no tienen utilidad terapéutica.

3260.4 Si ya no hay remedio, mejor no.

Evitar más sufrimiento

Respuestas que expresan el deseo de minimizar el sufrimiento $\quad 12 \quad 22.6 \quad$ Porque es solamente prolongar su agonía y la de las

tanto de los pacientes como de los familiares.

personas a su alrededor.

Dejar de ser carga

Cuando consideran que no tiene caso mantener la vida de alguien cuyo cuidado recae completamente en los demás.

$6 \quad 11.3$ Eso significaría estar al cuidado 100 \% de algún familiar.

Es antinatural

Cuando consideran que no debe utilizarse el procedimiento por tratarse de una prolongación artificial de la vida.

$3 \quad 5.7$ No es normal depender de una máquina,

Total

$53 \quad 100$

Participantes que sí están de acuerdo en la hidratación y alimentación artificiales

Es un deber

Expresan la obligación moral de alimentar e hidratar a alguien $\quad 36 \quad 69.2$ Porque todavía tiene vida, hay que hacer lo necesario. que no puede hacerlo por sí mismo.

Evitar inducir la muerte

Respuestas que consideran incorrecta una decisión que causaría la muerte del paciente.

1630.8 Porque no se puede dejar morir de hambre a alguien, a pesar de sus padecimientos.

Total

52100

Participantes que no están seguros si aprueban o no la hidratación y alimentación artificiales

Voluntad del paciente

Cuando indican que la decisión se debe basar en lo que el paciente haya expresado que querría.

1344.8 Depende qué haya decidido esta persona previamente a la enfermedad.

Depende de la voluntad de la familia y del paciente

Cuando creen que hay que tomar en cuenta tanto lo que haya expresado el paciente en su momento, como la opinión de los familiares a su cargo.

Incertidumbre

Respuestas que denotan dudas sobre si es adecuada o no la medida.

931.0 No lo sé, depende de si perjudicaría o le haría un beneficio.

Ambivalencia

Respuestas que denotan una valoración tanto positiva como negativa de la hidratación y alimentación artificiales.

$6 \quad 20.7$ Depende de lo decidido por la persona antes de llegar al punto de no recordar y de las resoluciones de la familia.

Total

13.4 Porque la persona debe seguir viviendo, pero no estoy totalmente de acuerdo porque uno debe morir en paz.

$29 \quad 100$

reciente reporte mundial de la Alzheimer's Disease International (95\%). ${ }^{8}$ En nuestro estudio, la mayoría de quienes no habían pensado en padecerla no conocía a nadie cercano con demencia.

Pensar en padecer demencia lleva a preguntarse sobre cómo vivir sin poder decidir, la dignidad y los

acuerdos con familiares antes de volverse incompetente. ${ }^{17}$ Algunas personas quieren actuar si la padecen, pero necesitan saber su diagnóstico. La mayoría de los participantes desearía conocerlo para prepararse para enfrentar la demencia, recibir ayuda terapéutica para mejorar su calidad de vida y decidir 
tratamientos futuros. Una minoría no quería o no estaba segura para no afligirse.

Puesto que en las demencias el deterioro cognitivo va aumentando, las personas diagnosticadas saben que perderán su capacidad para decidir. Los médicos deben informarles verazmente para que puedan ocuparse de su futuro, aunque los familiares pidan lo contrario. ${ }^{1,13}$ Sin embargo, de acuerdo con el reporte mundial de la Alzheimer's Disease International, $35 \%$ de los cuidadores en el mundo ha encubierto el diagnóstico. ${ }^{8}$ Si bien el control del enfermo sobre su vida involucra diversos aspectos (financieros, testamentarios), este estudio se enfocó a la atención médica. Los pacientes deben comunicar qué quieren en el futuro; aunque los médicos y los familiares buscan lo mejor para el paciente, sus valores pueden llevarlos a decidir intervenciones contrarias al enfermo.

La mitad de los participantes había comunicado a sus familiares su voluntad sobre tratamientos futuros a través de pláticas; muy pocos mediante un DVA oficial que permite rechazar tratamientos que prolongan la vida cuando, además de padecer demencia, una enfermedad amenaza la vida. Aunque el DVA se piensa para el estado avanzado de demencia, podría aplicarse en la etapa moderada si así lo decidiera la persona.

Se exploró la opinión sobre la alimentación e hidratación artificiales en pacientes con demencia que no pueden comer ni decidir. La frecuencia de respuestas de acuerdo y desacuerdo fue parecida. Como la alimentación tiene un fuerte valor social, muchos familiares sienten que no pueden dejar morir a su paciente, por lo que la alimentación artificial es frecuentemente solicitada por los familiares y también decidida por médicos, a pesar de haberse reportado que la alimentación enteral no beneficia a pacientes con demencia avanzada: no aumenta la supervivencia y puede causar daños (aspiración y desarrollo de úlceras). ${ }^{18} \mathrm{Al}$ estar afectadas las estructuras neurobiológicas en la etapa muy avanzada de la enfermedad, no se experimenta sed ni hambre ni sufrimiento asociado. ${ }^{19}$

Las razones de quienes estuvieron en desacuerdo con dar alimentación e hidratación artificiales coinciden con la evidencia médica de no estar indicada la alimentación parenteral y que sería futilidad terapéutica; además, consideraron que no darla evita más sufrimiento, se deja de ser una carga y que es antinatural. Quienes opinaron de esta forma podrían estar influidos por la idea de llegar a encontrarse en esa situación y no querer prolongar el sufrimiento de los familiares ni ser una carga. La principal razón para estar de acuerdo con la alimentación artificial fue considerar un deber alimentar a quien no puede hacerlo por sí mismo, argumento que coincide con la jerarquía católica que considera la alimentación y la hidratación como cuidados normales y que suspenderlas equivale a una eutanasia. La otra razón fue no inducir la muerte del paciente, idea que supone que siempre es mejor vivir más y se relaciona con el principio de la santidad de la vida que prohíbe causar la muerte..$^{20} \mathrm{La}$ principal explicación de quienes no estaban seguros fue que debía ser una decisión del paciente, lo cual refuerza la importancia de la voluntad anticipada y su doble función: que las personas confíen que su voluntad va a respetarse cuando ya no puedan expresarla y facilitar a los familiares las decisiones por ellos.

Reconociendo la utilidad de la voluntad anticipada, hay controversias de su uso en pacientes con demencia avanzada:

- Numerosas personas consideran más importante no vivir con demencia que prevenir el sufrimiento o la indignidad unos meses al final de la vida. No quieren ser dependientes ni una carga. Sin otra enfermedad que amenace su vida, cuyos tratamientos podrían rechazar para morir, se ven obligados a vivir. ${ }^{14}$

- Algunas personas no solo temen vivir con demencia, sino que su voluntad anticipada no sea respetada en la institución médica, como de hecho ha sucedido.

- Puede pasar que una persona documente su voluntad anticipada para no vivir en un estado avanzado de demencia y al llegar a él no sufra e, incluso, disfrute algunas experiencias. ${ }^{21}$

Se ha distinguido entre valores críticos que expresan intereses personales, como la idea de una vida digna, y los puramente experenciales. ${ }^{22} \mathrm{Al}$ escribir un DVA debería aclararse que se desea rechazar tratamientos (y morir), aún si uno parece estar contento, ${ }^{21}$ porque lo fundamental es no vivir cuando uno ha olvidado quién es. Esto plantea el dilema ético de si debe prevalecer la voluntad de la persona que era competente sobre la vida de la persona incompetente actual, problema que algunos filósofos han Ilamado "el problema de otra persona". ${ }^{23}$ Queda mucho por discutir para decidir cómo responder al interés de las personas de tener control sobre su vida si padecieran EA u otras demencias a través del DVA.

\section{Conclusiones}

Este estudio confirma el interés de un grupo de adultos maduros de tomar decisiones anticipadas ante la 
posibilidad de padecer demencia, la importancia de seguir investigando el tema para responder a este interés, resolver algunos dilemas éticos y promover el DVA.

Los hallazgos deben interpretarse dentro del contexto de sus limitaciones y sugerencias para estudios futuros. Primero, la muestra estuvo constituida por personas con un nivel educativo de licenciatura 0 superior; una muestra con diferentes escolaridades permitiría que fuera representativa de la población mexicana. Segundo, se exploró el porcentaje de participantes que habían comunicado su voluntad sobre futuros tratamientos y la opinión sobre decisiones que son legales en nuestro país. En futuros estudios sería interesante preguntar la opinión sobre la muerte asistida y la decisión de dejar de comer y beber que se ha documentado en otros países.

\section{Conflicto de intereses}

Las autoras declaran que no hay conflicto de intereses potencial con respecto a la investigación.

\section{Financiamiento}

No se recibió apoyo financiero para la investigación.

\section{Responsabilidades éticas}

Protección de personas y animales. Las autoras declaran que los procedimientos seguidos se conformaron a las normas éticas del comité de experimentación humana responsable y de acuerdo con la Asociación Médica Mundial y la Declaración de Helsinki.

Confidencialidad de los datos. Las autoras declaran que siguieron los protocolos de su centro de trabajo sobre la publicación de datos de pacientes.

Derecho a la privacidad y consentimiento informado. Las autoras obtuvieron el consentimiento informado de los pacientes o sujetos referidos en el artículo. Este documento obra en poder de la autora de correspondencia.

\section{Bibliografía}

1. D'Hyver C. Ética y Alzheimer. Es un dilema. En: Rivero-Serrano O, coordinador. Reflexiones sobre dilemas éticos en la práctica médica. Tomo I. México: Universidad Nacional Autónoma de México; 2018.

2. Brewer C. O, let me not get Alzheimer's sweet heaven! Reino Unido: Skycraoer Publications Limited; 2019.

3. Velázquez-López G. Síndromes demenciales. En: De la Fuente JR, Heinze G. Salud mental y medicina psicológica. Tercera edición. México: McGraw Hill; 2017.

4. Organización Mundial de la Salud [Internet]. Suiza: Demencia; 2019.

5. Gutiérrez-Robledo LM, Arrieta-Cruz I. Demencias en México: la necesidad de un plan de acción. Gac Med Mex. 2015;151:667-673.

6. Hernández A, Torres S. La necesidad de cuidados del paciente con Alzheimer y la respuesta social organizada. En: Gutiérrez-Robledo LM, García-Peña MC, Roa-Rojas PA, Martínez-Ruiz A, editores. La enfermedad de Alzheimer y otras demencias, como problema nacional de salud. México: Intersistemas/Consejo Nacional de Ciencia y Tecnología; 2017.

7. Volhard T, Jessen F, Kleineidam L, Wolfsgruber S, Lanzerath D, Wagner M, et al. Advance directives for future dementia can be modified by a brief video presentation on dementia care: An experimental study. PLoS One. 2018;13:e0197229.

8. World Alzheimer Report 2019: attitudes to dementia. Alzheimer's Disease International; 2019.

9. Reitz C. Genetic diagnosis and prognosis of Alzheimer's disease: challenges and opportunities. Expert Rev Mol Diagn. 2015;15:339-348.

10. Hickman RA, Faustin A, Wisniewski T. Alzheimer disease and its growing epidemic risk factors, biomarkers, and the urgent need for therapeutics. Neurol Clin. 2016;34:941-953.

11. Reitz C, Mayeux R. Alzheimer disease: epidemiology, diagnostic criteria, risk factors and biomarkes. Biochem Pharmacol. 2014;88:640-651.

12. AMGEN [Internet]. EE. UU.: World's largest Alzheimer's survey reveals most adults believe a cure will be developed in their lifetime; 2020 .

13. Piers R, Albers G, Gilissen J, de Lepeleire J, Steyaert J, van Mechelen, et al. Advance care planning in dementia: recommendations for healthcare professionals. BMC Palliat Care. 2018;17:88.

14. Steinbock B, Menzel PT. Advance directives for refusing life-sustaining treatment in dementia. Hastings Cent Report. 2018;48:S75-S79.

15. González-Huerta I, Gutiérrez-Soriano J, Álvarez-del Río A. Decisiones médicas sobre el final de la vida en pacientes con enfermedad de Alzheimer. México: Fontamara; 2017.

16. Gutiérrez-Robledo LM, García-Peña MC, Roa-Rojas PA, Martínez-Ruiz A, editores. La enfermedad de Alzheimer y otras demencias, como problema nacional de salud. México: Intersistemas/Consejo Nacional de Ciencia y Tecnología; 2017.

17. Detering KM, Hancock AD, Reade MC, Silvester W. The impact of advance care planning on end-of-life care in elderly patients: randomized controlled trial. BMJ. 2010;340:c1345.

18. Ying I. Artificial nutrition and hydration in advanced dementia. Can Fam Physician. 2015;61:245-248.

19. Trujillo-de los Santos Z. Cuidados paliativos. Arch Neurocytes (Mex). 2016;21:125-130.

20. Sumner LW. Physician-assisted death. EE. UU.: Oxford University Press; 2017.

21. Volicer L. Fear of dementia. JAMDA. 2016;17:875-878.

22. Dworkin R. Life's dominion. EE. UU.: Vintage Books; 1994

23. Degrazia D. Advance directives, dementia, and "The someone else problem". Bioethics. 1999;13:374-391. 